\title{
Simulation of insulin regimen and glucose profiles in Type 1 Diabetic Patient
}

\author{
Simona Panunzi*, Alessandro Borri*, Pasquale Palumbo ${ }^{\dagger}$, Levente Kovács ${ }^{\ddagger}$ and Andrea De Gaetano* \\ *BiomatLab, Istituto di Analisi dei Sistemi ed Informatica, Consiglio Nazionale delle Ricerche, Rome, Italy \\ ${ }^{\dagger}$ Istituto di Analisi dei Sistemi ed Informatica, Consiglio Nazionale delle Ricerche, Rome, Italy \\ $\ddagger$ John von Neumann Faculty of Informatics, Óbuda University, Budapest, Hungary
}

\begin{abstract}
A composite model, describing the glucose/insulin dynamics following daily food administration and insulin injections in Type 1 Diabetes Mellitus patients is presented. Three daily meals have been simulated, food intake representing four different types of foodstuffs, along with three rapid-acting insulin injections and one long-acting insulin injection. Three different scenarios (depending on whether food intake and/or administration times were fixed or random) were hypothesized: simulations show a very realistic time-course for both glucose and insulin dynamics over long (20 days) and short (one day) time periods.
\end{abstract}

\section{INTRODUCTION}

Type 1 Diabetes Mellitus (T1DM) is a form of diabetes that mainly results from the autoimmune destruction of $\beta$-cells, the pancreatic cells that produce, store and release insulin [1]. In healthy individuals, insulin secretion from pancreatic $\beta$-cells is stimulated in response to meals and is reduced to a low basal rate during the post-absorptive state. The main role of insulin is to regulate carbohydrate and fat metabolism in the body, promoting glucose uptake from tissues so that plasma glucose levels are maintained within a narrow range.

In T1DM, hyperglycemia (in the absence of naturally occurring insulin) can be controlled with exogenous insulin, commonly administered by subcutaneous injections at periodic intervals several times per day, mostly in correspondence of meals (though other options exist, such as insulin pumps). In order to best mimic insulin release in healthy subjects, insulin regimens often foresee the use of short-acting (e.g. regular human insulin) or rapid-acting (e.g. insulin lispro [2] or insulin aspart) insulin, administered within 15 minutes before a meal or immediately after a meal, along with the use of intermediate- or long-acting [3] insulin [4]. This approach is often referred as multiple daily injection (MDI) insulin therapy. The pre-meal short- or rapid-acting insulin addresses insulin requirements associated with the carbohydrate content of meals, while the longer-acting insulin addresses basal insulin requirements, i.e. the amount of insulin required to maintain glycaemic control between meals and during the overnight period. With this kind of therapeutic plan three to five injections per day are required. Alternative therapeutic schemes foresee twice-daily injection of mixtures of shortand longer-acting insulins (either self-prepared or ready-made, according to fixed ratio mixtures).

In any case, the objective is the optimal control in order to reduce hyperglycaemia, which is associated with long- term microvascular damage, blindness, renal impairment and peripheral neuropathy [5]. Another necessary objective is to reduce the risk of hypoglycemia, which represents the most common adverse effect associated with insulin-based therapies: severe hypoglycemia is extremely unpleasant, can determine seizures and may even cause death.

The aim of the present work is that of simulating, by a modeling approach, both real-life insulin therapy schemes and real-life (composite) meals, determining the resulting daily plasma glucose profiles. The model presented combines two sub-models: a recently developed simple mathematical model for the interpretation of data from an Oral Glucose Tolerance Test (OGTT) [6] and a complementary model representing rapid- and long-acting insulin injections. Therefore, while the original OGTT/meal model included a representation of spontaneous insulin secretion, in the present work the equations expressing the pancreatic response to glucose stimuli are replaced by equations describing exogenous insulin kinetics.

Simulations were run for a time sufficient to reach regime profiles under all simulated scenarios. Results related to the last day from each scenario are presented and compared.

\section{Models And Methods}

\section{A. The composite Glucose-Insulin Model}

A block diagram of the whole model is shown in Fig. 1. It consists of two sub-models. The first sub-model describes glucose dynamics in 5 main compartments, corresponding to four differential equations, a set of "stomach" compartments (each of them representing a different foodstuff type), and one algebraic equation. The second sub-model describes insulin kinetics: it is composed of three differential equations corresponding to the fast and slow insulin depot compartments and to the (unique) plasma insulin compartment.

1) The Glucose dynamics sub-model: The glucose submodel is a modified version of an already published model [6]. It differs by the introduction of different "stomach" compartments in parallel, in order to take into account the heterogeneous foodstuff composition of real meals. Each "stomach" compartment is therefore categorized by a different glycemic index, that is by the speed at which the glucose content in the food passes to jejunum and is then made available. In order to simplify the physiological analysis, the caloric contribution of non-carbohydrate foodstuffs has been represented in terms of the quantity of glucose which they eventually spare. In this 
sense, while it is actually possible for some protein to be converted to glucose itself (gluconeogenesis), fats essentially compete with glucose for entry into the Krebs (citric acid) cycle, thus determining a glucose sparing effect which is responsible, at least in part, for the insulin resistance deriving from high-fat diets (Randles cycle).

The equations of the sub-model are as follows:

$$
\begin{aligned}
& \frac{d S_{i}(t)}{d t}=-k_{j s} S_{i}(t), \quad S_{i}(0)=p_{i} D_{j}, \\
& i=1 . . . n, j=1,2,3 \\
& \frac{d J(t)}{d t}=\sum_{i=0}^{n} k_{j s_{i}} S_{i}(t)-k_{g j} J(t)-k_{r j} J(t), J(0)=0 \\
& \frac{d R(t)}{d t}=k_{r j} J(t)-k_{l r} R(t), R(0)=0 \\
& \frac{d L(t)}{d t}=k_{l r} R(t)-k_{g l} L(t), L(0)=0 \\
& \frac{d G(t)}{d t}=-k_{x g} G(t)-k_{x g i} I(t) G(t)+G_{P R O D}(t)+ \\
& +f\left[\frac{k_{g j} J(t)+k_{g l} L(t)}{V_{g} B W}\right], G(0)=G_{b}
\end{aligned}
$$

where

$$
G_{P R O D}(t)=T_{1 g} e^{-\lambda_{1 g} G(t)}+T_{2 g i} e^{-\lambda_{2 g} G(t) I(t)}
$$

and from the steady state conditions

$$
\begin{gathered}
G_{P R O D}(0)=\left(k_{x g}+k_{x g i} I_{b}\right) G_{b} \\
T_{1 g}=\frac{G_{P R O D}(0)-T_{2 g i} e^{-\lambda_{2 g} G_{b} I_{b}}}{T_{1 g} e^{-\lambda_{1 g} G_{b}}}
\end{gathered}
$$

Details about the above sub-model are reported in [6]. Briefly, $\mathrm{G}$ represents plasma glucose concentrations, $S_{i}, J$ and $L$ are glucose-equivalent amounts in several "stomach" compartments, in the jejunum and ileum, following a meal intake whose foodstuffs are indexed by $i$ and are in proportion of $p_{i}$. The $\mathrm{R}$ compartment is a delay compartment necessary to describe the transit of the glucose load through the intestinal lumen. Stomach glucose dynamics is described by (1). The elimination (emptying) terms depend on glucose-equivalent amounts, where $k_{j s_{i}}$ is the glucose transfer rate from "stomach" $i$ to the jejunum. From the jejunum glucose passes to the ileum through a delay compartment $\mathrm{R}$, and is absorbed with two potentially different transfer rate constants $\left(k_{g j}\right.$ and $\left.k_{g l}\right)$.

\begin{tabular}{|c|c|c|}
\hline Parameter & Description & Value \\
\hline \multicolumn{3}{|c|}{ GLUCOSE-SUB-MODEL } \\
\hline$G_{b}[m M]$ & $\begin{array}{l}\text { basal plasma glucose } \\
\text { concentration immediately } \\
\text { before glucose administration }\end{array}$ & 6.9 \\
\hline$D_{j}[\mathrm{mmol}]$ & $\begin{array}{l}\text { dose of glucose } \\
\text { administered at meal } \mathrm{j}\end{array}$ & $\begin{array}{l}417 \\
1389 \\
972\end{array}$ \\
\hline$p_{i}[\#]$ & proportion of foodstaff type $i$ & table III \\
\hline$V_{g}\left[L k^{-1}\right]$ & apparent glucose distribution volume & 0.19 \\
\hline$B W[k g]$ & weight & 65 \\
\hline$k_{j s_{i}}\left[\mathrm{~min}^{-1}\right]$ & $\begin{array}{l}\text { glucose transfer rate from } \\
\text { stomach i to jejunum }\end{array}$ & $\begin{array}{l}0.1,0.05 \\
0.025,0.01\end{array}$ \\
\hline$k_{g j}\left[\min ^{-1}\right]$ & $\begin{array}{l}\text { glucose transfer rate from jejunum } \\
\text { to plasma }\end{array}$ & 0.042 \\
\hline$k_{r j}\left[\min ^{-1}\right]$ & $\begin{array}{l}\text { glucose transfer rate from jejunum } \\
\text { to delay compartment }\end{array}$ & 0.09 \\
\hline$k_{l r}\left[\min ^{-1}\right]$ & $\begin{array}{l}\text { glucose transfer rate from the delay } \\
\text { compartment to ileum }\end{array}$ & 0.06 \\
\hline$k_{g l}\left[\min ^{-1}\right]$ & $\begin{array}{l}\text { glucose transfer rate from } \\
\text { ileum to plasma }\end{array}$ & 0.27 \\
\hline$k_{x g}\left[\min ^{-1}\right]$ & $\begin{array}{l}\text { insulin independent first order } \\
\text { glucose elimination rate }\end{array}$ & 0.001 \\
\hline$k_{x g i}\left[\min ^{-1} p M^{-1}\right]$ & $\begin{array}{l}\text { insulin dependent second order } \\
\text { glucose elimination rate }\end{array}$ & $5 \times 10^{-5}$ \\
\hline$T_{1 g}\left[\mathrm{~min}^{-1} m M\right]$ & $\begin{array}{l}\text { maximal rate of liver glucose } \\
\text { production (in plasma concentration } \\
\text { units) as dependent only } \\
\text { on glycemia }\end{array}$ & determined \\
\hline$T_{2 g i}\left[\min ^{-1} m M\right]$ & $\begin{array}{l}\text { maximal rate of liver glucose } \\
\text { (in plasma concentration units) } \\
\text { production as dependent on both } \\
\text { glycemia and insulinemia }\end{array}$ & 0 \\
\hline$\lambda_{1 g}\left[m M^{-1}\right]$ & $\begin{array}{l}\text { rate of decay of liver glucose } \\
\text { production with increasing glycemia }\end{array}$ & 0.58 \\
\hline$\lambda_{2 g i}\left[m M^{-1} p M^{-1}\right]$ & $\begin{array}{l}\text { rate of decay of liver glucose } \\
\text { production with increasing } \\
\text { glycemia and insulinemia }\end{array}$ & 0 \\
\hline$f[\#]$ & $\begin{array}{l}\text { fraction of bioavailable glucose from } \\
\text { gastrointestinal tract }\end{array}$ & 0.80 \\
\hline
\end{tabular}

Equation (5) describes glucose dynamics, with the first term on the right-hand side representing spontaneous glucose elimination rate and with the second term representing glucose tissue uptake due to insulin effect. $k_{x g i}$, the insulin-dependent glucose elimination rate, represents an index of insulin sensitivity. The Hepatic Glucose Output (HGO) described in (6) is dependent on circulating plasma glucose and insulin. Liver
TABLE I

MODEL PARAMETER DESCRIPTION AND VALUES

\section{INSULIN-SUB-MODEL}

\begin{tabular}{lll}
\hline$D_{k S}[\mathrm{pmol}]$ & quantity of injected slow insulin & table 11 \\
\hline$D_{j F}[\mathrm{pmol}]$ & quantity of injected fast insulin & table 11 \\
\hline \hline$V_{i}\left[\mathrm{Lkg}^{-1}\right]$ & apparent insulin distribution volume & 0.72 \\
\hline$I_{b}[\mathrm{pM}]$ & $\begin{array}{l}\text { basal plasma insulin concentration } \\
\text { immediately before glucose } \\
\text { administration }\end{array}$ & 130 \\
& $\begin{array}{l}\text { first order slow insulin } \\
\text { elimination rate }\end{array}$ & 0.0006 \\
\hline$k_{s i}\left[\mathrm{~min}^{-1}\right]$ & $\begin{array}{l}\text { first order fast insulin } \\
\text { elimination rate }\end{array}$ & 0.0115 \\
\hline$k_{f i}\left[\mathrm{~min}^{-1}\right]$ & $\begin{array}{l}\text { first order insulin } \\
\text { elimination rate }\end{array}$ & 0.02 \\
\hline$k_{x i}\left[\mathrm{~min}^{-1}\right]$ & bioavailability of fast insulin & 0.65 \\
\hline \hline$b[\mathrm{\#}]$ & & \\
\hline
\end{tabular}

glucose production is suppressed and glycogen-synthesis is enhanced in the presence of high plasma glucose and insulin concentrations. The first term in (6) describes net HGO as only dependent on plasma glucose levels, while the second term captures the response of the liver in the presence of high insulin concentrations. The last term of (5) represents glucose 


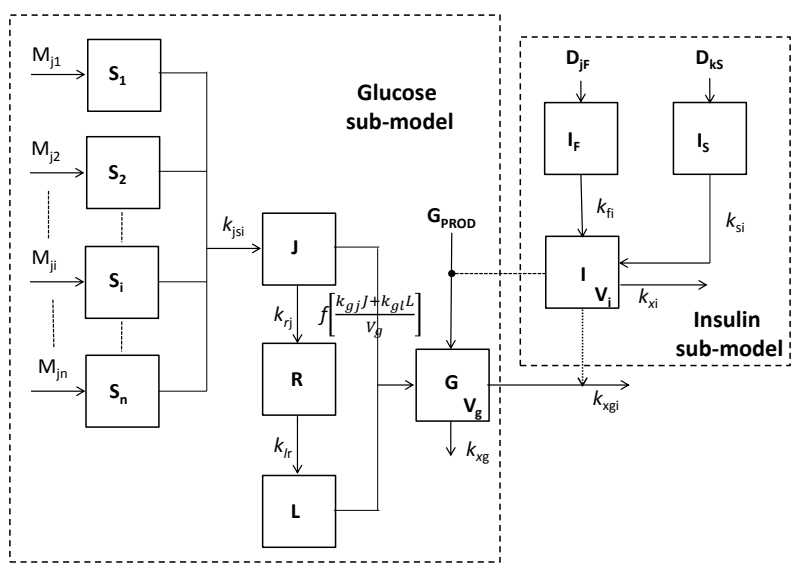

Fig. 1. Block diagram of the model. Schematic representation of the two sub-models. $M_{j i}$ represents the administered foodstuff $i$ at time $j$ and $S_{i}$ the respective glucose quantity in the Stomach. $J, R$ and $L$ is for the glucose content in the jejunum, in a delay compartment and in the ileum. $G$ indicates the glucose concentration in plasma. The Insulin sub-model is composed of three compartments: the slow-acting insulin depot $I_{S}$, the fast-acting insulin depot $I_{F}$, while $D_{k S}$ and $D_{j F}$ the respective insulin doses. Compartment $I$ is the plasma insulin concentration and $V_{i}$ the corresponding apparent distribution volume. Continuous lines represent entry or exit fluxes while dotted ones stimulation (arrows) or inhibition (black circles) mechanisms.

appearance into plasma due to meals, which goes through the "stomach" compartments and is absorbed from the small bowel. Since not all of the administered glucose amount is effectively absorbed, this term is multiplied by a fraction of absorption $f$. Table I reports description of model parameters as well as the values used in the simulations.

2) The Insulin dynamic sub-model: It describes the kinetics of exogenous insulin injections with the following equations:

$$
\begin{gathered}
\frac{d I_{S}(t)}{d t}=-k_{s i} I_{S}(t)+\sum_{k} \delta\left(t-t_{k}\right) D_{k S}, I_{S}(0)=0 \\
\frac{d I_{F}(t)}{d t}=-k_{f i} I_{F}(t)+\sum_{j} \delta\left(t-t_{j}\right) b D_{j F}, I_{F}(0)=0 \\
\frac{d I(t)}{d t}=\frac{k_{f i} I_{F}(t)+k_{s i} I_{S}(t)}{V_{i} B W}-k_{x i} I(t), I(0)=I_{b}
\end{gathered}
$$

where $I_{S}$ and $I_{F}$ represent the amounts of slow-acting and fast-acting insulin in the subcutaneous depot. Injection dose $D_{j F}$ of fast insulin occurs at time $t_{j}$, in correspondence of daily meal $j$ for $j=1,2,3$. The slow dose insulin $D_{k S}$ is administered once daily before bed rest at time $t_{k} . I$ is the serum insulin concentration, whereas $k_{s i}$ and $k_{f i}$ are the rate transfer constants from the slow and fast subcutaneous depots to the serum compartment. Parameter $k_{x p}$ is the elimination rate from plasma, $V_{i}$ is the apparent distribution volume, $b$ is the bio-availability of fast acting insulin ( 0.65 in this case), and $\delta$ is the Dirac delta, by means of which the succession of insulin injections is represented in the differential equations.

\section{B. Simulation approach}

All simulations are based on the real therapeutic schedule adhered to a 51 year-old female patient with T1DM. The therapy is reported in Table II; it is based on the use of a type of rapid-acting insulin (insulin lispro) administered at the time of each meal, along with the use of a long-acting insulin (insulin glargine), administered around 10:00 PM.

Table III reports the meal compositions. We have hypothesized a "basal" daily caloric intake of $2000 \mathrm{Kcal}$, divided into 3 meals per day (300 Kcal mean caloric intake breakfast, 1000 Kcal lunch, $700 \mathrm{Kcal}$ dinner). As 1 gr of glucose corresponds to $3.74 \mathrm{Kcal}$, the amount of administered glucose-equivalent is about equal to $75 \mathrm{gr}$ at breakfast, $250 \mathrm{gr}$ at lunch and 175 gr at dinner. Each meal is hypothesized to be composed of four different foodstuffs: simple sugars (foodstuff type A, e.g. orange juice), complex carbohydrates (type B, e.g. bread or potatoes), protein (type C, e.g. a lean steak) and fatty foods (type D, e.g. olive oil). Percentage composition of each meal, in terms of foodstuff types is reported in Table III.

TABLE II

THERAPEUTIC SCHEDULING

\begin{tabular}{c|c|c|c|c}
\hline & Breakfast & Lunch & Dinner & Before sleeping \\
\hline $\begin{array}{c}\text { Fasting insulin UI } \\
\text { pmol }\end{array}$ & 4 & 12 & 12 & \\
\hline $\begin{array}{c}\text { Slow Insulin UI } \\
\text { pmol }\end{array}$ & $(28000)$ & $(84000)$ & $(84000)$ & \\
\hline
\end{tabular}

TABLE III

COMPOSITION OF THE DAILY FOOD INTAKE

\begin{tabular}{c|c|c|c}
\hline & Breakfast & Lunch & Dinner \\
\hline $\begin{array}{c}\text { A: simple sugar } \\
\text { (i.e.orange juice) }\end{array}$ & $10 \%$ & $0 \%$ & $0 \%$ \\
\hline $\begin{array}{c}\text { B: complex carbohydrate } \\
\text { (i.e.pasta, potatoes) }\end{array}$ & $60 \%$ & $40 \%$ & $10 \%$ \\
\hline $\begin{array}{c}\text { C: protein } \\
\text { (i.e.steak) }\end{array}$ & $20 \%$ & $40 \%$ & $50 \%$ \\
\hline $\begin{array}{c}\text { D: fatty food } \\
\text { (i.e.fries, cheese) }\end{array}$ & $10 \%$ & $20 \%$ & $40 \%$ \\
\hline
\end{tabular}

Three simulation scenarios have been considered each running for 20 days in order to achieve a stable regime. In the first scenario ("basal" scenario) each meal occurs at exactly the same time and in exactly the same quantity over all simulated days: breakfast is always given at 8:00 AM, lunch at 1:00 PM and dinner at 8:00 PM. The sum of the gramms of administered glucose corresponds to the "basal" caloric intake.

In the second scenario, while the proportional meal composition (among the several types of foodstuff) and the administration times remains constant and unchanged, the administered glucose content at each meal is a random realization from a truncated normal distribution centered on the mean quantity of glucose (administered in the "basal" scenario) and with a standard deviation of $20 \%$ of the mean value. Low and high limits of the distribution were set to $\pm 60 \%$ of the mean value.

In the third scenario, besides having random quantities, randomness also occurs in the times at which meals are 
administered, while proportional meal composition remains constant (reflecting the gastronomic choices of the subject: always cappuccino, pastry and jam at breakfast, a big plate of pasta at lunch, meat and vegetables at dinner).

For each simulation the average glycemia, the maximal observed glycemia and the time necessary to reach normoglycemia after each meal for the last simulated day are recorded and compared among scenarios. All simulations were performed under Matlab v7.10.0 (R2010a) [7].

\section{RESULTS}

Fig. 2 reports the four foodstuff types of each daily meal for a complete run of 20 days. The top panel shows the simple sugars time-course (administered only once daily, at breakfast), with small spikes and fast dynamics. The following panels report complex carbohydrate, protein and fatty food intakes (all administered in different proportions, at each meal), showing taller spikes and slower dynamics. Fig. 3 reports plasma glucose (upper panel) and plasma insulin (bottom panel) concentrations following the meals and the insulin injections (central panels). As it can be seen, the equilibrium dynamics is quickly reached for all variables with fast dynamics, while only slow-acting insulin takes several days to reach its regime. Fig. 4-9 report the time-course of the simulated variables over the last simulated day, where stable regime has effectively reached for all threes scenarios. For each of scenarios 2 and 3 the 20 days run was simulated 2000 times, varying randomly the amounts of food intake (scenarios 2 and 3) and the food and injection administration times (only scenario 3). For these two scenarios $90 \%$ envelopes were reported along with the median of the obtained empirical distributions. In scenario 2 the envelopes determined by variable food intake appear narrow around their central trends (given reasonable standard deviations, low and high limits set for the generation of random realizations from truncated normal distributions), with the envelope around plasma glucose concentrations beings more appreciable (first panel in Fig. 7). In Fig. 8 (scenario 3), the wide envelopes are due to peaks are reached at different times as for variable meal times, even if the median trend results much lower that the higher percentile above all for the first panel (simple sugars). This is due to peaks are reached instantaneously and decrease is very fast due to the high elimination rate (which translates into fast dynamics). Percentiles at each time in scenario 3 must be interpreted with caution, since at each time the observed values derive from curves in different phases of the respective dynamics.

\section{Discussion}

While conceptually simple, the set of simulations reported may be of interest due to a combination of reasons. First of all, care has been taken in following the fate of glucose in plasma, as determined by a realistic representation of the accidental variations in lifestyle of real-life subjects. Actual therapeutic schedule of a real person has been used, populating the model with parameters taken from statistically accurate
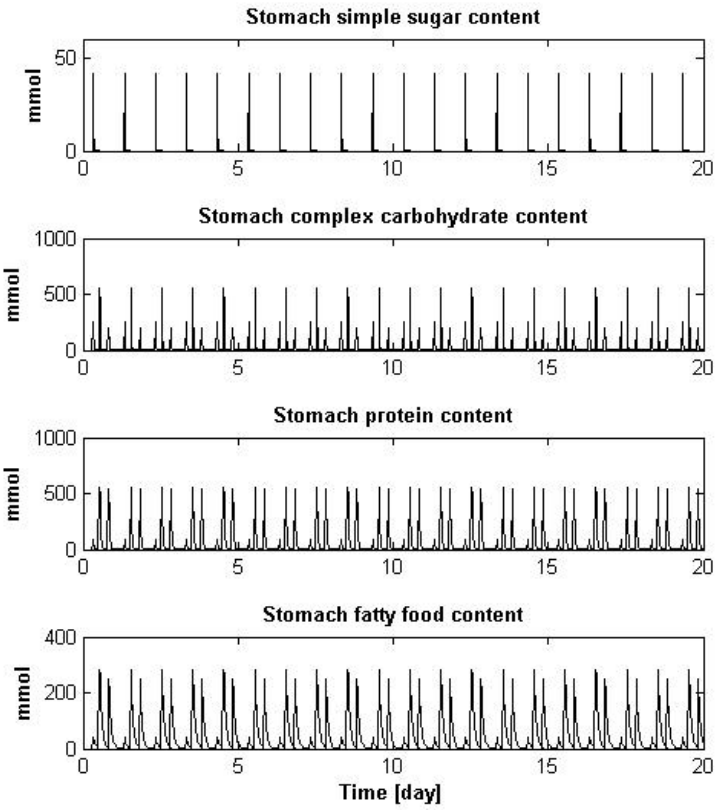

Fig. 2. Simulation of twenty days food intake for scenario 1
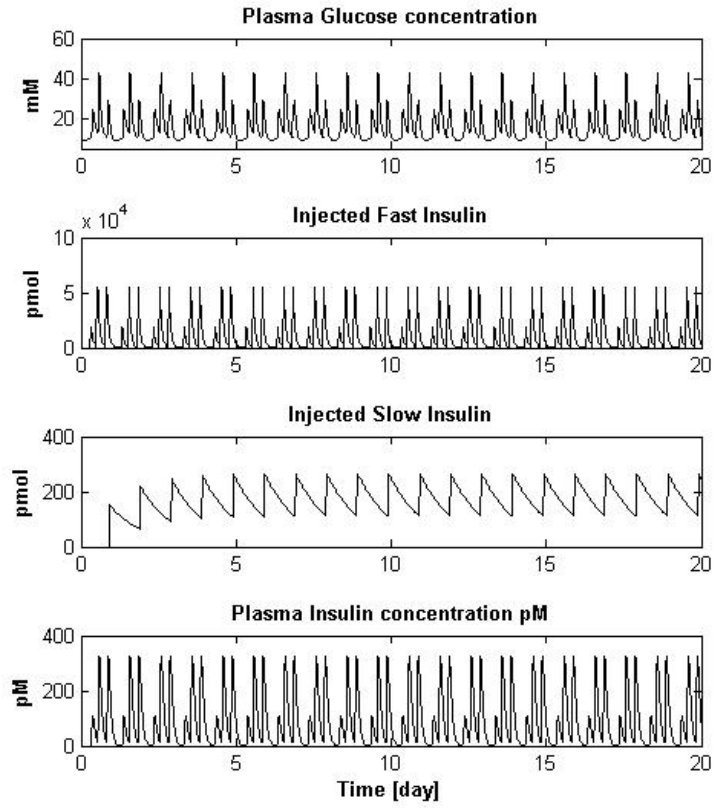

Fig. 3. Simulation of twenty days from scenario 1: glucose and insulin concentrations (first and last graph) along with quantity of slow (second graph) and fast (third graph) injected insulin for scenario 1

fitting procedures performed on large data sets, introducing a realistic variation in the composition of different meals.

The simple models used have been proven in several publications being robust, reliable and giving rise in particular 

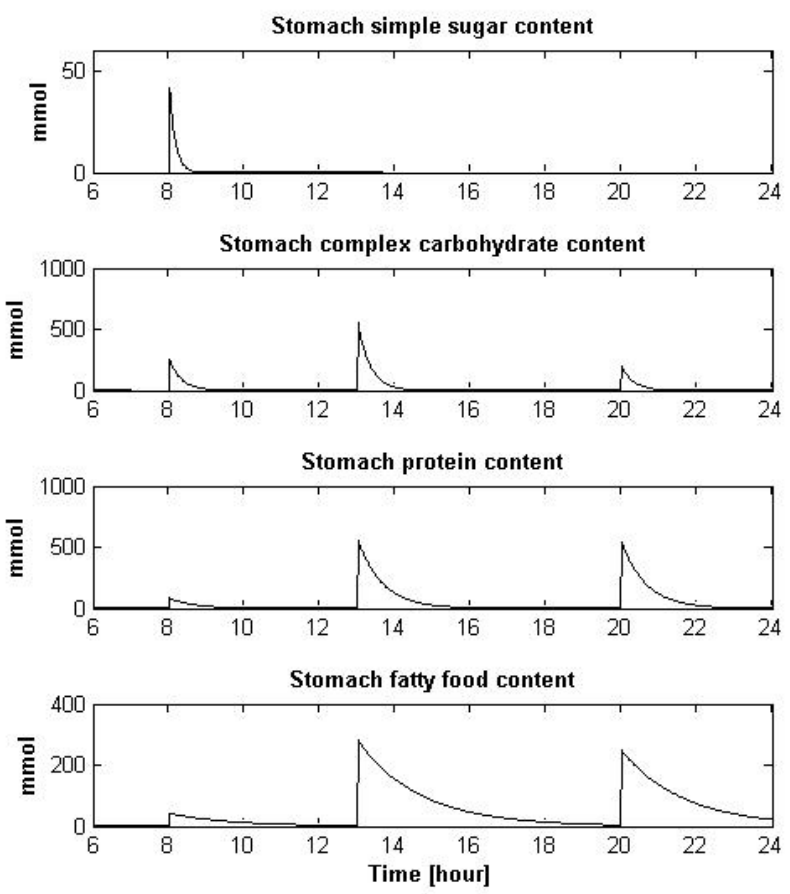

Fig. 4. Last day food intake for scenario 1
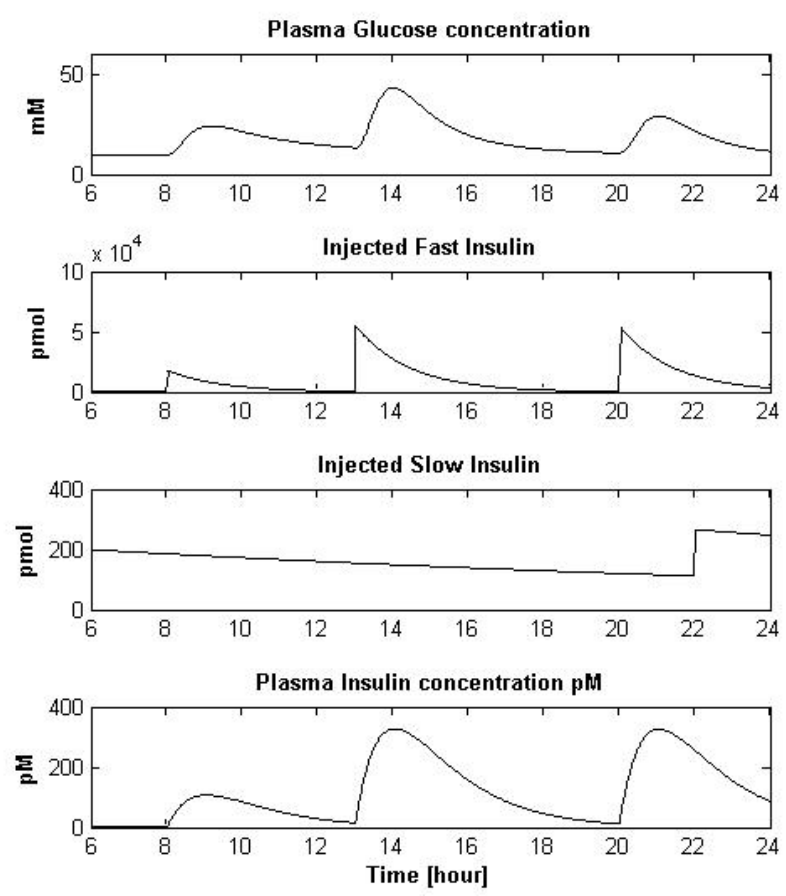

Fig. 5. Last day glucose and insulin concentrations (first and last graph) along with quantity of slow (second graph) and fast (third graph) injected insulin for scenario 1
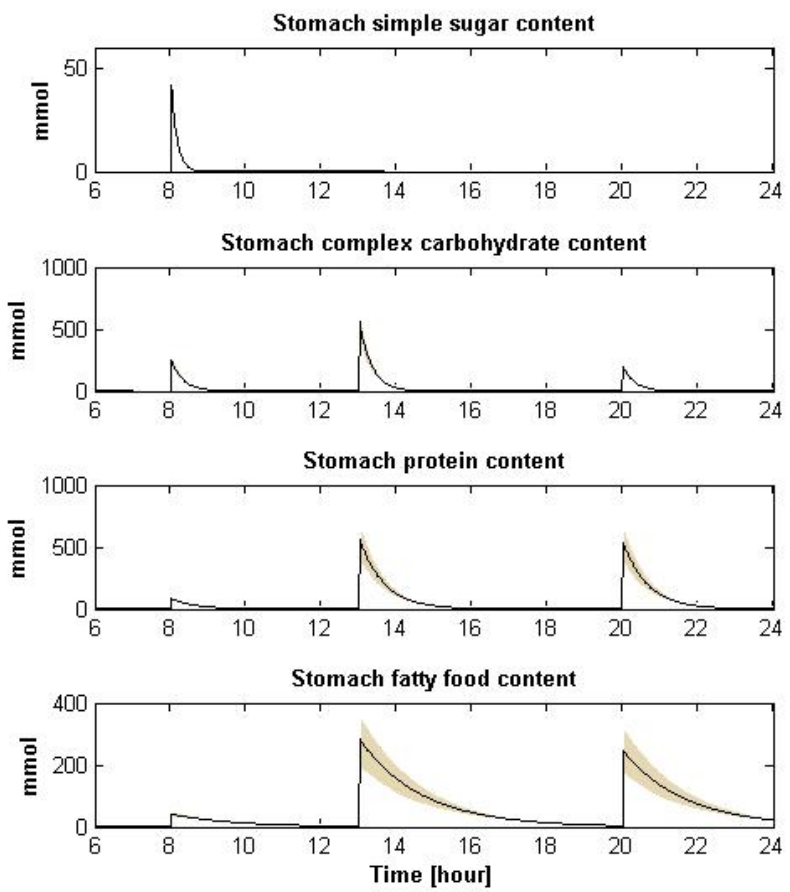

Fig. 6. Last day food intake for scenario 2
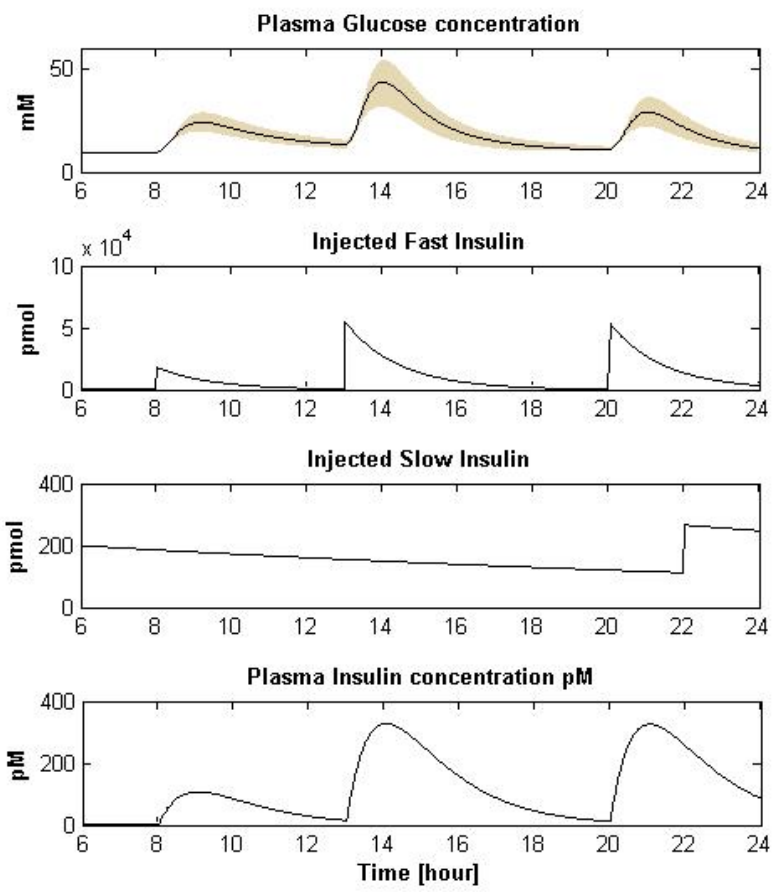

Fig. 7. Last day glucose and insulin concentrations (first and last graph) along with quantity of slow (second graph) and fast (third graph) injected insulin for scenario 2 

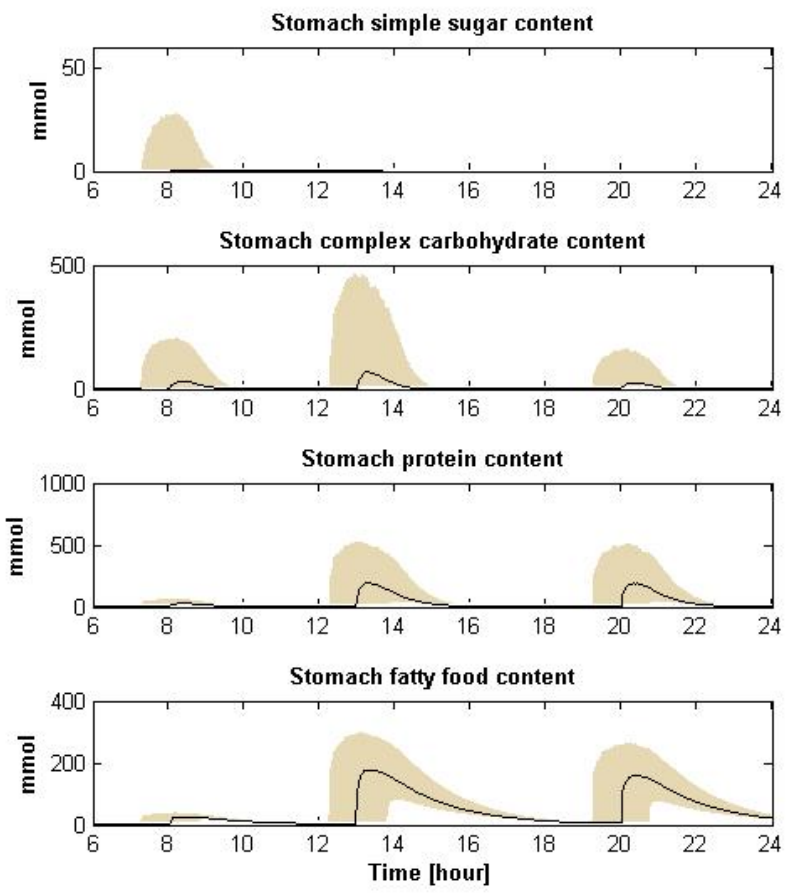

Fig. 8. Last day food intake for scenario 3
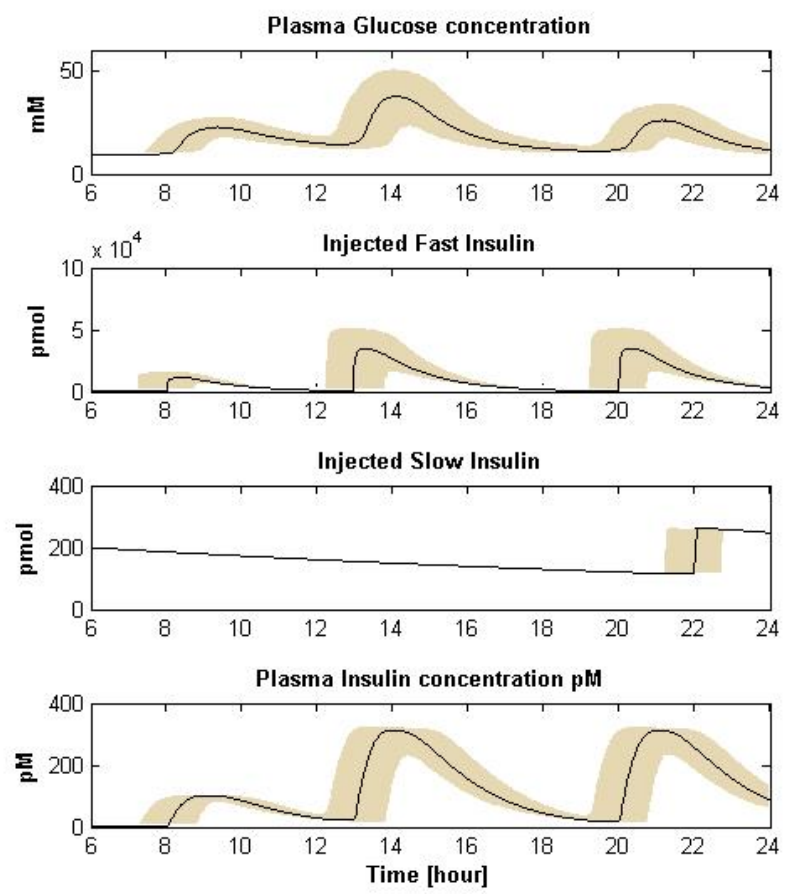

Fig. 9. Last day glucose and insulin concentrations (first and last graph) along with quantity of slow (second graph) and fast (third graph) injected insulin for scenario 3 to coherent physiological behavior and with estimated parameters within acceptable ranges of variability. The performed simulations reproduce glycemic profiles in accordance with expectations. Consequently, the aim of providing a reliable simulation platform for testing alternative therapeutic regimens or other control strategies, appears essentally met.

However, interpretation of the simulations produced suggests a rather interesting, unexpected phenomenon, which, if confirmed, may be of substantial importance in both diabetes care and in forecasting glycemic levels for control purposes. It appears that the $90 \%$ confidence envelopes are much wider when variability in the timing of food administration is allowed, rather when simple variability of food amount is present. In other words, it seems that glycemias can be predicted (hence controlled) much better if the patients allow themselfs some elasticity in how much food they consume, rather than when they are irregular in their eating times.

This potentially interesting conclusion needs to be confirmed by a thorough investigation of the relative sizes of the variance in food intake with respect to the variance in meal times over populations of interest. However, if it confirmes, the practical applications would be very concrete: in the effort to predict future glycemia, e.g. in a control environment, precise quantification of meal timing would be much more important than precise quantification of calorie content.

\section{CONClusion}

A robust, simple model has been constructed, able to replicate well the observed glycemic profiles in Type 1 Diabetes Mellitus patients in common real-life conditions, combining meals with fast and slow subcutaneous insulin administration.

\section{ACKNOWLEDGMENT}

L. Kovács is Bolyai Fellow of the Hungarian Academy of Sciences. The work was supported by the Hungarian GOP-1.1.1.-11-2012-0055 project and by the European Union TÁMOP-4.2.2.A-11/1/KONV-2012-0073 project.

\section{REFERENCES}

[1] A. D. Association, "Diagnosis and classification of diabetes mellitus," Diabetes Care, vol. 37, no. Supplement 1, Jan 2014.

[2] P. Roach and J. R. Woodworth, "Clinical pharmacokinetics and pharmacodynamics of insulin lispro mixtures," Clin Pharmacokinet, vol. 41, no. 13, pp. 1043-1057, 2002.

[3] sanofi-aventis U.S. LLC. (2014). [Online]. Available: http://www.drugs.com/pro/lantus.html

[4] D. B. Kelley, Medical management of type 1 diabetes.3rd ed. Alexandria (VA): American Diabetes Association, 1998.

[5] D. J. Goff, H. Gerstein, H. Ginsberg, W. Cushman, K. Margolis, R. Byington, J. Buse, S. Genuth, J. Probstfield, and D. G. SimonsMorton, "Prevention of cardiovascular disease in persons with type 2 diabetes mellitus: current knowledge and rationale for the action to control cardiovascular risk in diabetes (accord) trial," Am J Cardiol., vol. 99, no. (12A), pp. 4i-20i, Jan 2007.

[6] A. De Gaetano, S. Panunzi, A. Matone, A. Samson, J. Vrbikova, B. Bendlova, and G. Pacini, "Routine ogtt: a robust model including incretin effect for precise identification of insulin sensitivity and secretion in a single individual," PLoS One, vol. 29, no. 8(8), Aug 2013.

[7] MATLAB, version 7.10.0 (R2010a). Natick, Massachusetts: The MathWorks Inc., 2010. 